\title{
Quenching Time Estimates for Semilinear Parabolic Equations Controlled by Two Absorption Sources in Control System
}

\author{
DAI Xiaoqiang ${ }^{1}$, YANG Chao $^{2, *}$, HUANG Shaobin ${ }^{2}$ and WU Fei ${ }^{3}$ \\ ${ }^{1}$ Department of Electronic Information, Jiangsu University of Science \\ and Technology, Zhenjiang 212003, China. \\ 2 College of Computer Science and Technology, Harbin Engineering University, \\ Harbin 150001, China. \\ ${ }^{3}$ No.704 Institute of China Shipbuilding Industry Corporation (CSIC), \\ Shanghai 200000, China.
}

Received 15 January 2020; Accepted 6 February 2020

\begin{abstract}
This paper deals with the quenching solution of the initial boundary value problem for aclass of semilinear reaction-diffusion equation controlled by two absorption sources in control system and estimate upper bound and lower bound of the quenching time. We point that the number of absorption sources influences the time of quenching phenomenon. The solution can solve some boundary value problem in control system.
\end{abstract}

AMS Subject Classifications: 35A07, 35B50, 35K55

Chinese Library Classifications: O175.29

Key Words: Reaction-diffusion equation; Dirichlet boundary; quenching time; control system.

\section{Introduction}

The purpose of the present paper is to consider the quenching phenomenon for the initial boundary value problem (IBVP) of semilinear reaction-diffusion equation

$$
\begin{array}{ll}
u_{t}(x, t)-\Delta u(x, t)=(b-u(x, t))^{-p}+(b-u(x, t))^{-q} & \text { in } \Omega \times(0, T), \\
u(x, t)=0 & \text { on } \partial \Omega \times(0, T)
\end{array}
$$

*Corresponding author. Email addresses: daixiaoqiang@just.edu.cn (X. Q. Dai), yangchao_@hrbeu.edu.cn (C. Yang), huangshaobin@hrbeu .edu.cn (S. B. Huang), 13636616007@139.com (F. Wu) 


$$
u(x, 0)=u_{0}(x) \geq 0 \quad \text { in } \Omega,
$$

where $2<p<q, b=$ const $>0, \Omega \subset \mathbb{R}^{N}$ is a bounded domain, $\partial \Omega$ is its smooth boundary, and $u_{0}(x)$ is the nonnegative initial data in $C^{1}(\bar{\Omega})$ and $\sup _{x \in \Omega} u_{0}(x)<b$. We define $(0, T)$ to be the maximal existence time interval of the solution $u$ of (1.1)-(1.3) throughout the whole paper. The solution $u(x, t)$ of (1.1)-(1.3) has the following properties: $u(x, t)$ has twice continuous derivate in $x \in \Omega$ and once in $u(x, t)<b$ for all $t \in(0, T)$. Problem (1.1)-(1.3) represents an elastic membrane inside an idealized electrostatically actuated MEMS.

Definition 1.1. If $T=+\infty$, we say problem (1.1)-(1.3) admits a global solution. If $T<\infty$ and the solution $u(x, t)$ of problem (1.1)-(1.3) has a singularity

$$
\limsup _{t \rightarrow T} u(x, t)=b,
$$

then the solution $u(x, t)$ is the so-called quenching solution of problem (1.1)-(1.3), $T$ is the quenching time.

In 1975, Kwawarada [1] investigated the quenching phenomena firstly, formed the basis for further investigation by various authors [2]- [11]. Particularly, Boni and Bernard [7] studied a class of parabolic model with a single absorption source

$$
\begin{array}{ll}
u_{t}=L u+r(x)(b-u)^{-p}, & (x, t) \in \Omega \times(0, T), \\
u=0, & (x, t) \in \partial \Omega \times(0, T), \\
u(x, 0)=u_{0}(x) \geqslant 0, & x \in \Omega .
\end{array}
$$

Further, they obtained the quenching phenomena of problem (1.4)-(1.6) and estimated the quenching time. Also, they clearly demonstrated that the absorption source term has an pronounce affect on the quenching phenomenon for the nonlinear reaction diffusion equation. $\mathrm{Xu}$ [9] investigated initial boundary value problem (1.4)-(1.6) for nonlinear parabolic differential equations with several combined nonlinearities and carries out numerical experiments. Selcuk [10] and Ozalp [11] showed quenching phenomenon occurs on the singular boundary conditions. The present paper focuses on the solution of the same type of equation with two absorption sources, which are both positive. We change the exponents of both two absorption sources such that the two terms have a large enough gaps in the sense of growth order, in order to reveal and compare the importance of the two factors acting on the behavior of the quenching phenomena, which are the number of the absorption source terms and the exponents of these terms. The result$s$ obtained in the preset paper suggests the dominant influence of the exponents of the absorption terms comparing the number of them, which is not only different from the classical heat equation with nonlinear power-type external force [12, 13], but also different from the nonlinearities and their corresponding behaviours and affects in other models [14-30]. 
The paper consists of following sections: In Section 2, we show the local solution of problem (1.1)-(1.3) by approximation method. In Section 3, we prove the quenching phenomenon and estimate the quenching time by the maximum principle and corresponding ODE theory.

\section{Local existence}

Firstly, we prove that the solution locally exists.

Theorem 2.1. Problem (1.1)-(1.3) admits a unique local solution on $\Omega \times(0, T)$, where $T<\infty$.

Proof. Let $V(x, y, t)$ be the fundamental solution of problem

$$
\begin{array}{ll}
h_{t}-\triangle h=0 & \text { in } \quad \Omega \times(0, \infty), \\
h=0 & \text { on } \quad \partial \Omega \times(0, \infty),
\end{array}
$$

which is defined on $\bar{\Omega} \times \bar{\Omega} \times(0, \infty)$ and satisfies

$$
\begin{aligned}
& V(x, y, t)>0, \quad(x, y, t) \in \Omega \times \Omega \times(0, \infty), \\
& \int_{\bar{\Omega}} V(x, y, t) \mathrm{d} y \leqslant 1 .
\end{aligned}
$$

Then problem (1.1)-(1.3) has the solution of the following form

$$
u(x, t)=\int_{\Omega} V(x, y, t) u(y, 0) \mathrm{d} y+\int_{0}^{t} \int_{\Omega} g(u(y, \tau)) V(x, y, t-\tau) \mathrm{d} y \mathrm{~d} \tau,
$$

where $(x, y, t) \in \Omega \times \Omega \times(0, T)$ and $g(u)=(b-u)^{-p}+(b-u)^{-q}$.

Next we construct the function sequence $\left\{u_{n}\right\}$ by putting

$$
\begin{aligned}
& u_{1}(x, t)=0, \\
& u_{n+1}(x, t)=\int_{\Omega} V(x, y, t) u(y, 0) \mathrm{d} y+\int_{0}^{t} \int_{\Omega} g\left(u_{n}(y, \tau)\right) V(x, y, t-\tau) \mathrm{d} y \mathrm{~d} \tau, \quad n \geq 0 .
\end{aligned}
$$

It is obvious that $u_{n}>0$ for all $n>1$, as $g(u)$ is increasing and $V(x, y, t)>0$. From the recurrence of (2.1), we can get $u_{n+1} \geqslant u_{n}$ in $\Omega \times(0, T)$.

Assume that $u_{0}(x) \leqslant b-2 \gamma$ and $u_{n} \leqslant b-\gamma$, where $\gamma$ is a positive number. We claim that $u_{n+1}$ has upper bounds, and $u_{n+1} \leqslant b-\gamma$ on a small time interval. From (2.1), we have

$$
u_{n+1} \leq(b-2 \gamma)+g(b-\gamma) \int_{0}^{T} \int_{\Omega} V(x, y, t-\tau) \mathrm{d} y \mathrm{~d} \tau
$$

As

$$
\lim _{t \rightarrow 0} \int_{0}^{t} \int_{\Omega} V(x, y, t-\tau) \mathrm{d} y \mathrm{~d} \tau=0
$$


there exists a small enough $T$ such that

$$
\int_{0}^{T} \int_{\Omega} V(x, y, t-\tau) \mathrm{d} y \mathrm{~d} \tau \leqslant \frac{\gamma}{g(b-\gamma)} .
$$

Combined (2.2) and (2.3), we have $u_{n+1} \leq b-\lambda$ for sufficient small T. Hence $\left\{u_{n}\right\}_{n \geqslant 1}$ is the increasing sequence and has the upper bound, i.e., $\left.u_{n}\right|_{n \geqslant 1} \leq b-\gamma$. From the monotone convergence theorem, there exists $\lim _{n \rightarrow \infty} u_{n}=u$ in $\Omega \times(0, T)$ satisfying

$$
u(x, t)=\int_{\Omega} V u(y, 0) \mathrm{d} y+\int_{0}^{t} \int_{\Omega} g(u(y, \tau)) V(x, y, t-\tau) \mathrm{d} y \mathrm{~d} \tau, \quad(x, t) \in \Omega \times(0, T) .
$$

\section{Quenching time}

In this section, we investigate the quenching phenomenon of the problem (1.1)-(1.3) and estimate the time of quenching.

For the initial datum, we define its supremum by taking $x=a \in \Omega$ as follows

$$
M=\sup _{x \in \Omega} u_{0}(x)=u_{0}(a) .
$$

Show the eigenvalue problem as follows

$$
\begin{array}{ll}
\lambda_{\delta} \varphi(x)+\Delta \varphi(x)=0, & x \in R(a, \delta), \\
\varphi(x)=0, & x \in \partial R(a, \delta), \\
\varphi(x)>0, & x \in R(a, \delta),
\end{array}
$$

where $R(a, \delta)=\left\{x \in \mathbb{R}^{N}:|x-a|<\delta\right\} \subset \Omega$ for $\delta>0$. Boni and Bernard [7] pointed that there exists a solution $\left(\varphi, \lambda_{\delta}\right)$ of problem (3.2)-(3.4) satisfying $0<\lambda_{\delta} \leqslant \frac{D}{\delta^{2}}$, where $D>0$ depends on the dimension $N$ and the upper bound of the coefficients of the operator $\Delta$. Further, we define $\int_{R(a, \delta)} \varphi \mathrm{d} x=1$.

Next, we show the main theorem.

Theorem 3.1. Assume that $K>0$ satisfies $\sup _{x \in \Omega} \frac{d u_{0}}{d x} \leq K$. Let $M>0$ and $E=K^{2} D b 2^{q}$. Then the quenching phenomenon occurs provided

$$
b-M<\min \left\{1,\left(\frac{1}{E}\right)^{\frac{3}{q+1}},\left(\operatorname{Kdist}(a, \partial \Omega)^{\frac{3}{q+1}}\right)\right\} .
$$

Further we estimate its quenching time $T$ as follows

$$
\int_{Q}^{b} \frac{\mathrm{d} u}{g(u)} \leqslant T \leqslant \frac{\left(b-M+(b-M)^{(q+1) / 3}\right)^{q+1}}{(q+1)\left(1-E(b-M)^{(2 q-1) / 3}\right)}
$$

where $g(u)=(1-u)^{-p}+(1-u)^{-q}$. 
Proof. Since $u_{0} \in C^{1}(\bar{\Omega})$, by the mean value theorem, there exists a $x_{0} \in R(a, \delta)$ satisfying

$$
u_{0}^{\prime}\left(x_{0}\right)=\frac{u_{0}(a)-u_{0}(x)}{\delta} .
$$

Taking $M=u_{0}(a), K=\sup _{x \in \Omega} u_{0}^{\prime}$, we have $K \geqslant \frac{M-u_{0}(x)}{\delta}$, that is $u_{0}(x) \geqslant M-K \delta$. If $\delta=\frac{1}{K}(b-$ $M)^{(q+1) / 3}$, we have

$$
u_{0}(x) \geqslant M-(b-M)^{(q+1) / 3} .
$$

Let $\phi(x, t)$ be a solution of the following IBVP

$$
\begin{cases}\phi_{t}-\Delta \phi=(b-\phi)^{-p}+(b-\phi)^{-q}, & (x, t) \in R(a, \delta) \times\left(0, T_{*}\right), \\ \phi=0, & (x, t) \in \partial R(a, \delta) \times\left(0, T_{*}\right), \\ \phi(x, 0)=u_{0}(x) \geqslant 0, & x \in R(a, \delta),\end{cases}
$$

where $T_{*}$ is the maximal existence time of $\phi(x, t)$. Since $\phi(x, 0)=u_{0}(x) \geqslant 0$ in $R(a, \delta)$, from the maximum principle, it follows that $\phi(x, t) \geq 0$ in $R(a, \delta) \times\left(0, T_{*}\right)$. We define $l(t)$ as follows

$$
l(t):=\int_{R(a, \delta)} \phi(x, t) \varphi(x) \mathrm{d} x, t \in\left[0, T_{*}\right) .
$$

Through using (3.2) and (3.7), we have the derivative of $l(t)$

$$
\begin{aligned}
l^{\prime}(t)= & \int_{R(a, \delta)} \varphi(x) \Delta \phi(x, t) \mathrm{d} x+\int_{R(a, \delta)}(b-\phi(x, t))^{-p} \varphi(x) \mathrm{d} x+\int_{R(a, \delta)}(b-\phi(x, t))^{-q} \varphi(x) \mathrm{d} x \\
= & \int_{R(a, \delta)} \phi(x, t) \Delta \varphi(x) \mathrm{d} x+\int_{R(a, \delta)}(b-\phi(x, t))^{-p} \varphi(x) \mathrm{d} x+\int_{R(a, \delta)}(b-\phi(x, t))^{-q} \varphi(x) \mathrm{d} x \\
=-\lambda_{\delta} l(t)+\int_{R(a, \delta)}(b-\phi(x, t))^{-p} \varphi(x) \mathrm{d} x & \\
& \quad+\int_{R(a, \delta)}(b-\phi(x, t))^{-q} \varphi(x) \mathrm{d} x, \quad t \in\left(0, T_{*}\right) .
\end{aligned}
$$

By Jensen's inequality, we have

$$
l^{\prime}(t) \geqslant-\lambda_{\delta} l(t)+(b-l(t))^{-p}+(b-l(t))^{-q} .
$$

Combined with $l(t) \in[0, b]$ for $0<t<T_{*}$ and

$$
0<\lambda_{\delta} \leqslant \frac{D}{\delta^{2}}=\frac{D K^{2}}{(b-M)^{(2 q+2) / 3}},
$$

we obtain that

$$
l^{\prime}(t) \geqslant(b-l(t))^{-q}\left(1+(b-l(t))^{q-p}-D K^{2} b(b-l(t))^{q}(b-M)^{-(2 q+2) / 3}\right) .
$$


From (3.6), we have

$$
\begin{aligned}
l(0) & =\int_{R(a, \delta)} u_{0}(x) \varphi(x) \mathrm{d} x \geq \int_{R(a, \delta)} M-(b-M)^{(q+1) / 3} \varphi(x) \mathrm{d} x \\
& =M-(b-M)^{(q+1) / 3} \int_{R(a, \delta)} \varphi(x) \mathrm{d} x=M-(b-M)^{(q+1) / 3},
\end{aligned}
$$

that is

$$
b-l(0) \leqslant b-M+(b-M)^{(q+1) / 3} \leqslant 2(b-M) .
$$

From (3.8) and (3.9), we have

$$
l^{\prime}(0) \geqslant \frac{1+(b-l(0))^{q-p}}{(b-l(0))^{q}}-\frac{D K^{2} b 2^{q}(b-M)^{(q-2) / 3}}{(b-l(0))^{q}},
$$

that is

$$
l^{\prime}(0) \geqslant \frac{1+(b-l(0))^{q-p}}{(b-l(0))^{q}}-\frac{E(b-M)^{(q-2) / 3}}{(b-l(0))^{q}}>0 .
$$

Next we prove

$$
l^{\prime}(t)>0, \quad t \in\left(0, T_{*}\right) .
$$

Arguing by contradiction, we suppose that $t_{1} \in\left(0, T_{*}\right)$ is the first time that $l^{\prime}(t)>0$ for $t \in\left[0, t_{1}\right)$ and $l^{\prime}\left(t_{1}\right)=0$. Then we see $l\left(t_{1}\right) \geqslant l(0)$, which means

$$
\begin{aligned}
0 & =l^{\prime}\left(t_{1}\right) \\
& \geqslant \frac{1+\left(b-l\left(t_{1}\right)\right)^{q-p}}{\left(b-l\left(t_{1}\right)\right)^{q}}-\frac{D K^{2} b\left(b-l\left(t_{1}\right)\right)^{q}(b-M)^{-(2 q+2) / 3}}{\left(b-l\left(t_{1}\right)\right)^{q}} \\
& \geqslant \frac{1+(b-l(0))^{q-p}}{(b-l(0))^{q}}-\frac{E(b-M)^{(q-2) / 3}}{(b-l(0))^{q}}>0
\end{aligned}
$$

which is a contradiction. Hence we have

$$
b-l(t) \leqslant b-l(0) \leqslant 2(b-M) .
$$

Further we obtain

$$
l^{\prime}(t) \geqslant \frac{1-E(b-M)^{(q-2) / 3}}{(b-l(t))^{q}} .
$$

By the direct calculation, one sees

$$
(b-l(t))^{q} \mathrm{~d} l(t) \geqslant\left(1-E(b-M)^{(q-2) / 3}\right) \mathrm{d} t .
$$

Integrating (3.10) from 0 to $T_{*}$ with respect to $t$ gives

$$
(b-l(0))^{q+1}(q+1)^{-1} \geqslant\left(1-E(b-M)^{(q-2) / 3}\right) T_{*},
$$


that is

$$
T_{*} \leqslant \frac{\left(b-M+(b-M)^{(q+1) / 3}\right)^{q+1}}{(q+1)\left(1-E(b-M)^{(q-2) / 3}\right)} .
$$

Since the right hand of (3.11) is bounded, we obtain that $\phi(x, t)$ quenches in a finite time. Using the maximum principle, it implies $u(x, t) \geqslant 0$ in $\Omega \times(0, T)$. And extending the estimate, we get

$$
u(x, t) \geqslant \phi(x, t), \quad(x, t) \in R(a, \delta) \times(0, \tilde{T}),
$$

for $\tilde{T}=\min \left\{T, T_{*}\right\}$, and

$$
T \leqslant T_{*} \leqslant \frac{\left(b-M+(b-M)^{(q+1) / 3}\right)^{q+1}}{(q+1)\left(1-E(b-M)^{(q-2) / 3}\right)} .
$$

In fact, assume that $T>T_{*}$, we see $\left\|u\left(x, T_{*}\right)\right\|_{\infty} \geqslant\left\|\phi\left(x, T_{*}\right)\right\|_{\infty}=b$, which contradicts the interval $(0, T)$ of the solution $u$. From the finite $T, u_{t}>0$, and $u<b$, we see that $u$ quenches in a finite time.

In the subsequence, we discuss an ODE problem to show the lower bound of $T$

$$
\begin{aligned}
& \frac{\mathrm{d} \eta(t)}{\mathrm{d} t}=g(\eta), \\
& \eta(0)=M,
\end{aligned}
$$

where $g(\eta)=(b-\eta)^{-p}+(b-\eta)^{-q}$ and $M=\sup _{x \in \Omega} u_{0}(x)<b$.

Assume that $s(x, t)=\eta(t)$ in $\bar{\Omega} \times\left[0, T_{k}\right)$, we have

$$
\begin{cases}s_{t}-\Delta s=(b-s)^{-p}+(b-s)^{-q}, & (x, t) \in \Omega \times\left(0, T_{k}\right), \\ s \geqslant 0, & (x, t) \in \partial \Omega \times\left(0, T_{k}\right), \\ s(0) \geqslant u_{0}(x), & x \in \Omega .\end{cases}
$$

By the maximum principle, one sees $0 \leqslant u \leqslant s=\eta(t),(x, t) \in \Omega \times\left(0, T_{k}\right)$.

Hence $g(u)>0$ implies

$$
\int_{M}^{\eta(t)} \frac{\mathrm{d} u}{g(u)}=t
$$

Assume that $T_{k}$ is the time such that $\lim _{t \rightarrow T_{k}} \eta(t)=b$. Then we see

$$
T_{k}=\int_{M}^{b} \frac{\mathrm{d} u}{g(u)}
$$

which implies that

$$
T \geqslant T_{k}=\int_{M}^{b} \frac{\mathrm{d} u}{g(u)} .
$$


However if $T_{k}>T$, then $\eta(T) \geqslant\|\phi(x, T)\|_{\infty}=b$, which contradicts the interval $\left(0, T_{k}\right)$ of the solution $\eta(t)$.

Combined with (3.12) and (3.13), we have the following estimate for $T$

$$
\int_{M}^{b} \frac{\mathrm{d} u}{g(u)} \leqslant T \leqslant \frac{\left(b-M+(b-M)^{(q+1) / 3}\right)^{q+1}}{(q+1)\left(1-E(b-M)^{(q-2) / 3}\right)} .
$$

\section{Authors' contributions}

Dr. Xiaoqiang Dai suggested the research topic and finished the main part of such research. Mr. Chao Yang wrote the main codes to simulated the solution and to verify the behavior of the quenching phenomena in the numerical way. Dr. Shaobin Huang checked all the arguments. Dr. Fei Wu conducted the corresponding experiments and the verification of the model and the special nonlinearities. All of them were involved in the work of revision and approved the final manuscript.

\section{Acknowledgement}

The authors thank Prof. Yanbing Yang and Dr. Mingyou Zhang for their constructive suggestions.

\section{References}

[1] Kawarada H., On solutions of the initial-boundary value problem for $u_{t}=u_{x x}+1 /(1-u)$. Publications of the Research Institute for Mathematical Sciences, 10 (1975), 729-736.

[2] Levine H.A., The phenomenon of quenching: A survey. North-Holland Mathematics Studies, New York, 110 (1985), 275-286.

[3] Levine H.A., Quenching, nonquenching and beyond quenching for solution of some parabolic equations. Ann. Mat. Pura Appl. 155 (1989), 243-260.

[4] Nabongo D., Boni T.K., Quenching time for some nonlinear parabolic equations. Analele ştiințifice ale Universităţii Ovidius Constanta, 16 (2008), 91-106.

[5] Phillips D., Existence of solution of quenching problems. Appl. Anal. 24 (1987), 253-264.

[6] Dai Q., Gu Y., A short note on quenching phenomena for semilinear parabolic equations. J. Differ. Equ. 137 (1997), 240-250.

[7] Boni T.K., Diby B.Y., Quenching time of solutions for some nonlinear parabolic equations with Dirichlet boundary condition and a potential. Annales Mathematicae et Informaticae, 35 (2008), 31-42.

[8] Acker A., Walter W., The Quenching Problem for Nonlinear Parabolic Differential Equations. Lect. Notes Math. 564 (2006), 1-12.

[9] Xu R., Jin C., Yu T., Liu Y., On quenching for some parabolic problems with combined powertype nonlinearities. Nonlinear Anal.-Real World Appl. 13 (2012), 333-339.

[10] Selcuk B., The Quenching behavior of a semilinear heat equation with a singular boundary outflux. Q. Appl. Math. LXXII (2014), 747-752. 
[11] Ozalp N., Selcuk B., The quenching behavior of a nonlinear parabolic equation with a singular boundary condition. Hacet. J. Math. Stat. 44 (3) (2015), 615-621.

[12] Xu R., Lian W., Niu Y., Global well-posedness of coupled parabolic systems. Sci. China-Math. DOI: https://doi.org/10.1007/s11425-017-9280-x

[13] Xu R., Wang X., Yang Y., Blowup and blowup time for a class of semilinear pseudo-parabolic equations with high initial energy. Appl. Math. Lett. 83 (2018), 176-181.

[14] Lian W., Xu R., Global well-posedness of nonlinear wave equation with weak and strong damping terms and logarithmic source term. Adv. Nonlinear Anal. 9 (2020), 613-632.

[15] Lian W., Xu R., Radulescu V.D., Yang Y., Zhao N., Global well-posedness for a class of fourth order nonlinear strongly damped wave equations. Adv. Calc. Var. DOI: https://doi.org/10.1515/acv-2019-0039

[16] Xu R., Wang X., Yang Y., Chen S., Global solutions and finite time blow-up for fourth order nonlinear damped wave equation. J. Math. Phys. 56 (6) (2018), 061503.

[17] Papageorgiou, Nikolaos S., Radulescu V.D., Nonlinear nonhomogeneous Robin problems with superlinear reaction term. Adv. Nonlinear Stud. 16 (2016), 737-764.

[18] Papageorgiou N.S., Radulescu V.D., Multiplicity of solutions for resonant Neumann problems with an indefinite and unbounded potential. Trans. Amer. Math. Soc. 367 (2015), 87238756.

[19] Bonanno G., Giovanni M.B., Radulescu V.D., Existence of three solutions for a nonhomogeneous Neumann problem through Orlicz-Sobolev spaces. Nonlinear Anal. 74 (2011), 4785-4795.

[20] Bonanno G., Giovanni M.B., Radulescu V.D., Quasilinear elliptic non-homogeneous Dirichlet problems through Orlicz-Sobolev spaces. Nonlinear Anal. 75 (2012), 4441-4456.

[21] Papageorgiou N.S., Radulescu V.D, Repovs D.D, Nonlinear analysis-theory and methods. Springer Monographs in Mathematics, Springer, Cham, 2019.

[22] Zhang Q., Radulescu V.D., Double phase anisotropic variational problems and combined effects of reaction and absorption terms. J. Math. Pures Appl. 118 (9) (2018), 159-203.

[23] Gazzola F., Weth T., Finite time blow up and global solutions for semilinear parabolic equations with initial data at high energy level. Differ. Integral Equ. 18 (2005), 961-990.

[24] Xu R.Z., Su J., Global existence and finite time blow-up for a class of semilinear pseudoparabolic equations. J. Funct. Anal. 264 (2013), 2732-2763.

[25] Xu R.Z., Wang X.C., Yang Y.B., Blowup and blowup time for a class of semilinear pseudoparabolic equations with high initial energy. Appl. Math. Lett. 83 (2018), 176-181.

[26] Di H.F., Shang Y.D., Peng X.M., Blow-up phenomena for a pseudo-parabolic equation with variable exponents. Appl. Math. Lett. 64 (2017), 67-73.

[27] Meyvaci M., Blow up of solutions of pseudoparabolic equations. J. Math. Anal. Appl. 352 (2009), 629-633.

[28] Bertsch M., Smarrazzo F., Tesei A., Pseudo-parabolic regularization of forwardbackward parabolic equations: a logarithmic nonlinearity. Anal. PDE, 6 (2013), 1719-1754.

[29] Di H.F., Shang Y.D., Zheng X.X., Global well-posedness for a fourth order pseudo -parabolic equation with memory and source terms. Discrete Contin. Dyn. Syst. Ser. B, 21 (3) (2016), 781-801.

[30] Xu R.Z., Su J., Global existence and finite time blow-up for a class of semilinear pseudoparabolic equations. J. Funct. Anal. 264 (2013), 2732-2763. 